\title{
BEL-1 Transactivator Responsive Sequences in the Long Terminal Repeat of Human Foamy Virus
}

\author{
OTTO ERLWEIN AND AXEL RETHWILM' \\ Institut fur Virologie und Immunbiologie der Universităt, Versbacher Str. 7, 97078 Würzburg, Germany
}

Received February 4, 1993; accepted lune 3, 1993

\begin{abstract}
Cis-regulatory elements in the long terminal repeat (LTR) of human foamy virus (HFV) were identified by using LTR mutants to transiently express the chloramphenicol acetyl-transferase gene after co-transfection with an expression plasmid for the virus bel-1 (transactivator) gene. The R.U5 region and an element in the $5^{\prime}$ U3 region were found to negatively influence HFV gene expression. The complete BEL-1 responsive region was mapped to extend from nucleotide position -471 to position -93 relative to the start of transcription. Within this region, three elements were identified that in the homologous or a heterologous (SV40) promoter context can, independently and irrespective of their orientation, act as targets for BEL-1. These elements are located between nucleotide positions $-413 /-378$, $-361 /-291$, and $-124 /-93$. The target elements do not share obvious sequence homologies. The mechanism of HFV transactivation appears to be novel among the complex retroviruses and is likely to involve, as yet, undiscovered cellular DNA binding factors. 1993 Academic Press, Inc.
\end{abstract}

\section{INTRODUCTION}

Human foamy virus (HFV) belongs to the retroviruses which regulate their gene expression by a transacting activator of viral transcription (Cullen, 1991). The viral bel-1 gene has been identified to encode for a $36 \mathrm{k}$ nuclear phosphoprotein that augments HFV long terminal repeat (LTR) directed transcription in a variety of mammalian and in avian cells (Rethwilm et al., 1991; Keller et al., 1991; Venkatesh et al., 1991, Venkatesh et al., 1993). Transcript mapping experiments revealed three mRNAs, two of which are multiply spliced, that may give rise to BEL-1 protein (Muranyi and Flögel, 1991). BEL- 1 is the only regulatory HFV protein which is essential for virus replication in vitro (Baunach et al., 1993). No further activity than the transcriptional effects of BEL-1 could be attributed to this protein (Baunach et al., 1993).

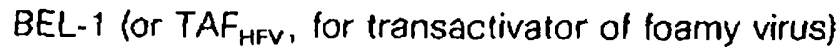
acts on a U3 DNA element of the viral LTR (Rethwilm et al., 1991; Keller et al., 1991; Venkatesh et al., 1991). The foamy virus $U_{3}$ regions are among the longest compared to other retroviruses; HFV U3 is 777 bp long. and the U3 regions of simian foamy virus (SFV) type 1 and 3 are 1296 and 1332 bp in length, respectively (Mergia et al., 1990; Renne et al., 1992).

\footnotetext{
1 To whom correspondence and reprint requests should be addressed.
}

In previous studies on the mapping of BEL-1 responsive elements in HFV U3, the BEL-1 target region was located upstream of positions -94 (Keller et al., 1991) and -62 (Venkatesh et al., 1991) respectively, relative to the start of transcription. A more definitive analysis has been performed on the TAF response region of SFV-1 and SFV-3. Two regions in the SFV-1 LTR beiween positions -1196 and -880 and between -403 and -125 were identified to respond to $T A F_{S F V_{-1}}$; the latter element was also found to act as an inducible enhancer in an orientation independent manner in the context of a heterologous promoter (Mergia et al., 1992). In the SFV-3 LTR, two regions $(-637 /-496$ and $-496 /-180$, respectively) that confer $\mathrm{TAF}_{\mathrm{SFV}-3}$ responsiveness to a heterologous promoter, irrespective of their orientation, have been mapped (Renne et al:, 1993). HFV and SFV U3 regions are highly divergent compared to $R$ and $U 5$ sequences (Renne et al., 1992). This is reflected by a low degree of HFV and SFV TAF amino acid homology compared to the env and pol proteins (Mergia et al., 1991; Renne et al., 1992). Thus, TAF $_{\text {SFV-1 }}$ does not stimulate the HFV LTR (Mergia et al., 1992). Enhancement of SFV-3 LTR activity by TAF SFV-1 has been described (Renne et al., 1993) which might reflect the closer relatedness of these two viruses (Renne et al., 1992). However, TAF $_{\mathrm{SFv} .3}$ does not stimulate reporter gene expression driven by the SFV-1 LTR (Renne et al., 1993).

While the results of these experiments suggest a highly specific mechanism of foamy virus transactiva- 


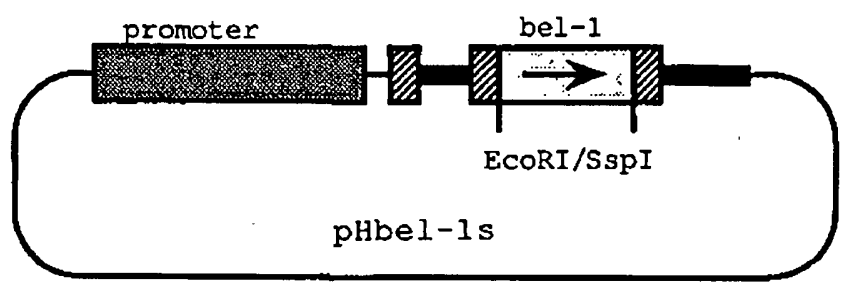

Fig. 1. pHbel-1s expression vector, which was co-transfected with the indicator plasmids in all experiments. The bel- 1 coding sequence was cloned as an Sspl fragment derived from plasmid pHSRV (Rethwilm et al., 1990) into the EcoRl site in the third exon of the rabbit $\beta$-globin gene of pKCR3 (O'Hare et al., 1981) in which the SV40 promoter was replaced by the mouse $\mathrm{MHC}$ class I $\mathrm{H}-2 \mathrm{~K}^{\mathrm{b}}$ enhancer/ promoter (Kimura et al., 1986). Intron and polyadenylation sequences are indicated as bold lines.

tion, TAF $_{\mathrm{HFV}}$ and $\mathrm{TAF}_{\mathrm{SFV}}$ proteins were found to augment lentivirus LTR directed gene expression, which may also indicate some pleiotropic effects of foamy TAF proteins (Keller et al., 1991; Mergia et al., 1992). The human immunodeficiency virus type 1 (HIV-1) U3 element between positions $-158 /-118$ was found to be essentiell for transactivation by TAF $_{\mathrm{HFV}}$ (Lee et al., 1992; Keller et al., 1992). Within this element is contained a nonamer motif which shares eight out of nine nucleotides with an HFV U3 motif between positions $-135 /-127$. This sequence has therefore been considered as the BEL-1 responsive element (BRE).

Since knowledge of the TAF target site(s) in the HFV LTR is crucial in elucidating the mechanism of HFV specific transactivation, we constructed a series of LTR mutants and assayed their responsiveness to
BEL- 1 in both homologous and heterologous promoter contexts. Our results support the view that the mechanism of foamy virus transactivation is novel among complex retroviruses.

\section{MATERIALS AND METHODS}

\section{Plasmid constructions}

All DNA manipulations were done by established molecular cloning techniques (Sambrook et al., 1989). Recombinant plasmids were characterized by dideoxy sequencing (Sanger et al., 1977).

The BEL-1 expression plasmid, pHbel-1s, was constructed by inserting the bel- 1 coding sequence as an Sspl fragment derived from pHSRV (Rethwilm et al., 1990 ) into the ECORI site of pH2EV atter filling in with Klenow enzyme. PH2EV is a derivative of the pKCR eukaryotic expression vector (O'Hare et al., 1981). The vector contains the mouse $\mathrm{MHC}$ class I $\mathrm{H}-2 \mathrm{~K}^{\mathrm{b}}$ promotor (Kimura et al., 1986) and was a generous gift from I. Horak, Würzburg (1. Horak, unpublished). This plasmid is shown in Fig. 1. pHbel-1a is a non-expressing control plasmid harboring the bel-1 gene in reverse orientation with respect to the promoter.

p5'LTR and p5'LTRinv were the source of all further LTR mutants and were generated by excising the Kpn// Narl fragment, spanning the complete $5^{\prime} L T R$, from the infectious molecular clone pHSRV (Rethwilm et al., 1990), treatment with Klenow enzyme, and insertion

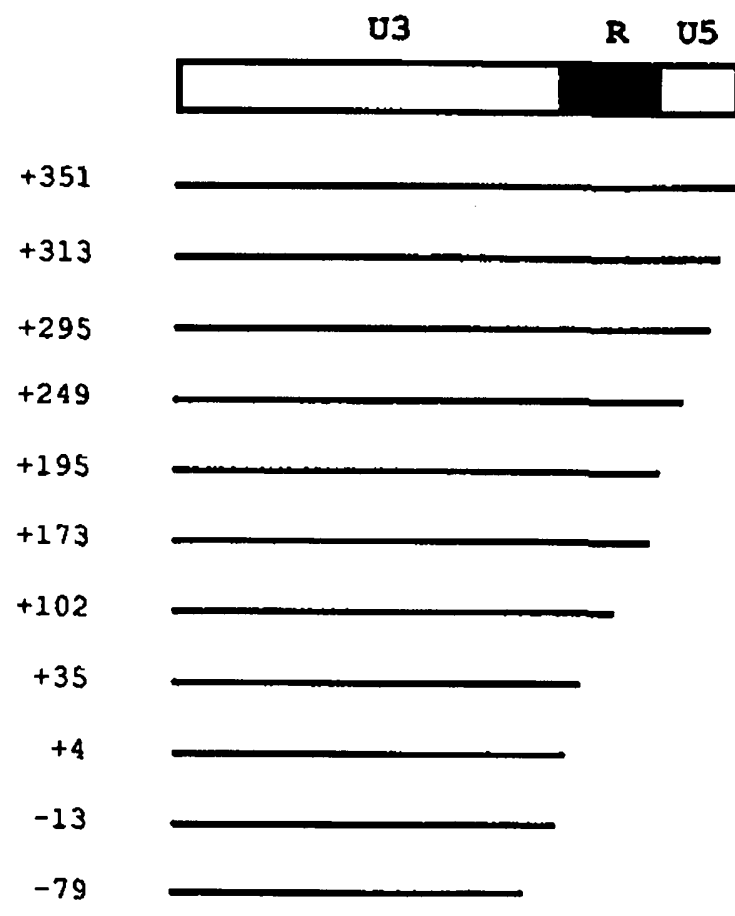

\begin{tabular}{|c|c|c|}
\hline- TAF & + TAF & $\begin{array}{c}\text { fold } \\
\text { activation }\end{array}$ \\
\hline 1.0 & 10.1 & 10 \\
\hline 0.7 & 61.7 & 88 \\
\hline 0.3 & 38.9 & 129 \\
\hline 0.3 & 69.1 & 230 \\
\hline 0.3 & 86.7 & 289 \\
\hline 0.3 & 94.4 & 314 \\
\hline 0.3 & 84.3 & 281 \\
\hline 0.3 & 92.9 & 310 \\
\hline 0.3 & 92.9 & 310 \\
\hline 0.8 & 84.9 & 106 \\
\hline 0.5 & 2.8 & 6 \\
\hline
\end{tabular}

FIG. 2. Positive effect of nested deletions of the R-U5 region on HFV LTR promotor activity. Assay conditions: $50 \mu \mathrm{g}$ of protein lysate was incubated for $60 \mathrm{~min}$. 


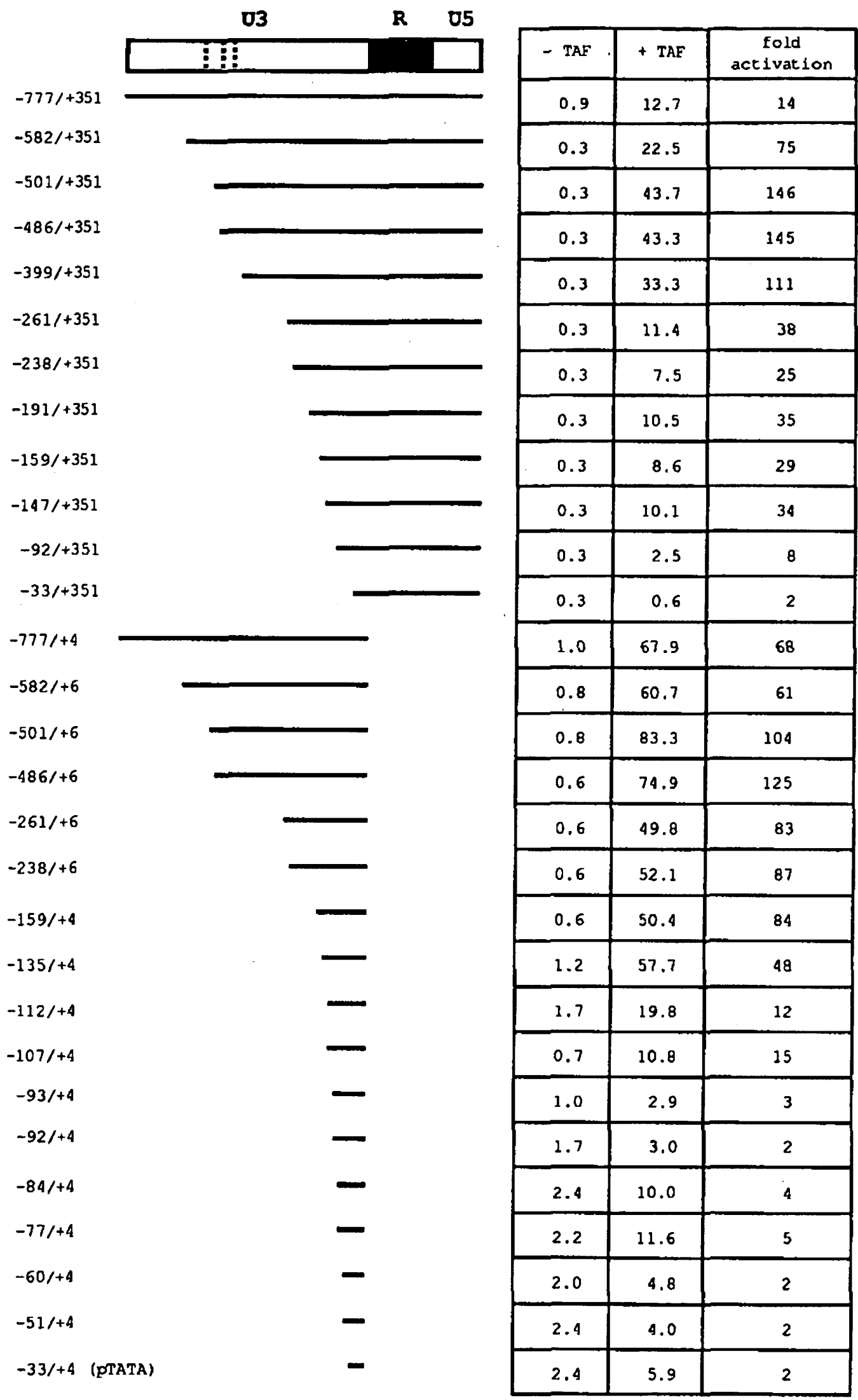

FIG. 3. The BRE in the HFV U3 region is located upstream of nucleotide position -93 relative to the start of transcription. Nested $5^{\prime}$ deletions of the HFV LTR were analyzed to determine the $3^{\prime}$ border of the BRE. The dotted vertical lines in the LTR represent the three binding sites for the transcription factor AP-1. Assay conditions: $50 \mu \mathrm{g}$ protein of lysates from cells transfected with constructs bearing the R-U5 region and $25 \mu \mathrm{g}$ from cells transfected with constructs bearing only U3 sequences was incubated for $60 \mathrm{~min}$. 


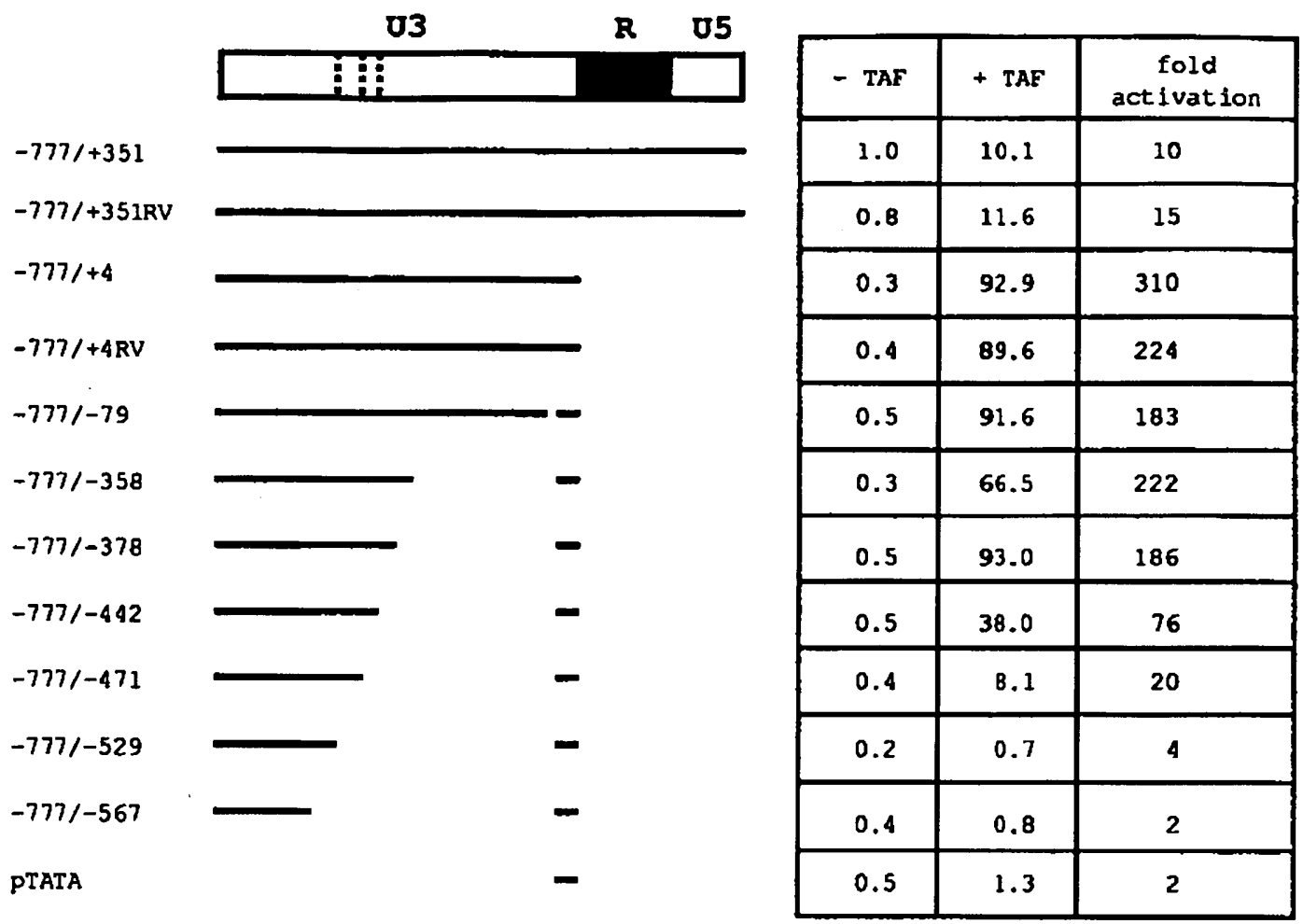

FIG. 4. The BRE is located downstream of nucleotide position -471 . Nested $3^{\prime}$ deletions of the U3 region were analyzed to determine the $5^{\prime}$ border of the BRE. The dotted lines represent the three binding sites for the transcription factor AP-1. Assay conditions: $50 \mu \mathrm{g}$ protein was incubated for $60 \mathrm{~min}$.

into the Smal site of the pUC19 vector in either orientation.

Deletion mutagenesis of R-U5 sequences from the $3^{\prime}$ end was performed by Bal31 digestion of EcoRI linearized $p 5$ 'LTR, Klenow treatment and religation with a Bgll linker. BamHI/Bg/ll fragments were excised from the resulting plasmids and inserted into the chloramphenicol acteyl-transferase (CAT) expression vector pocat-Bg (Rethwilm et al., 1990), resulting in the constructs shown in Fig. 2.

Similarly deletion mutagenesis of U3 sequences from the $5^{\prime}$ end was carried out by Bal31 digestion of EcoRI linearized p5'LTRinv. p5'cat constructs obtained this way are depicted in Fig. 3. For removal of the R-U5 region in these constructs a Bal31 $3^{\prime}$ digestion product deleting sequences up to base +4 (start of transcription is +1 ) was used, alternatively R-U5 was deleted with Sacl which cuts at position +6 and in the pUC polylinker.

To facilitate deletion mutagenesis of the U3 region from the 3' end, an EcoRV site was introduced immediately upstream of the TATA box at position -34 relative to the start of transcription by in vitro mutagenesis, using the oligonucleotide 5'-CTTATATACGATATCCATCATTCTTAAAAC-3' (mutated nucleotides are in bold face) after subcloning a Hindlli/Xbal fragment of p5'LTR into the $13 \mathrm{mp} 19$ vector. The mutated Hindlll/ $X b a l$ fragment was then substituted for the respective fragment of $p 5^{\prime}$ cat $(-777 /+351)$ and $p 5^{\prime}$ cat $(-777 /+4)$ leading to $p 5^{\prime}$ cat $(-777 /+351) \mathrm{RV}$ and $\mathrm{p} 5{ }^{\prime} \mathrm{cat}(-777)$ $+4) R V$, respectively. $3^{\prime}$ U3 deletions were introduced into p5'cat $(-777 /+4) \mathrm{RV}$ by cutting with EcoRV and appropriate restriction enzymes in $\mathrm{U} 3$ and religation after Klenow enzyme treatment. Alternatively, HindIII (position -362) cut plasmid was digested with Bal31 and religated after EcoRV cleavage so that only the truncations in the $5^{\prime}$ direction from the Hindlll site became effective. Plasmids obtained this way are shown in Fig. 4.

The pTATA $(-33 /+4)$ vector was created by deleting the U3 region upstream of the TATA box as a Bg/ll/ EcoRV fragment from p5'cat $(-777 /+4) \mathrm{RV}$ and religation with a BgAl linker. LTR fragments or chemically synthezised oligonucleotides introduced into this vector are depicted in Figs. 5-8.

The enhancer-less vector $\mathrm{pA}_{10}$ cat (Laimins et al., 1984) was used to test LTR motifs for enhancer function in a heterologous (SV40) promoter context. DNA fragments of the HFV LTR, or chemically synthesized and annealed oligonucleotides, were introduced into the $B g / l$ site $5^{\prime}$ to the CAT gene. These constructs are shown in Fig. 9.

\section{Transfections and CAT-assays}

Baby hamster kidney cells (BHK-21) and dog thymus Cf2Th fibroblasts were obtained from Dr. NeumannHaefelin (Freiburg, Germany) and the ATCC, respec- 


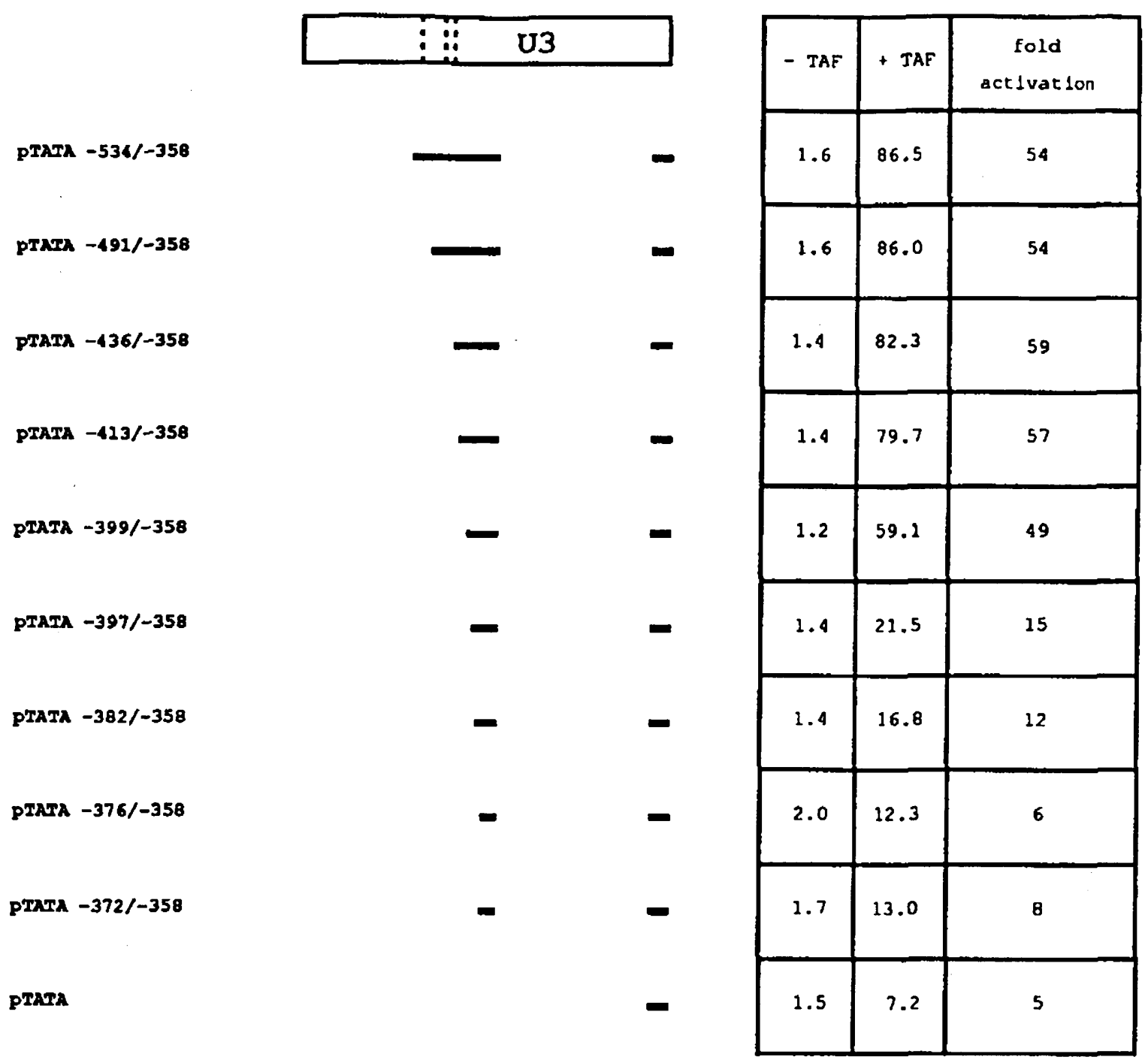

FIG. 5. BRE $I$ is located between nucleotide positions -413 and -358 . Nested 5 deletions of the $-534 /-358$ sequence of $U 3$ introduced into the pTATA $(-33 /+4)$ vector were assayed to map BRE I. The dotted lines represent the AP- 1 binding sites. Assay conditions: $50 \mu \mathrm{g}$ protein were incubated for $60 \mathrm{~min}$.

tively. Both cells are susceptible to HFV infection (Rethwilm et al., 1990; and our unpublished observation). Cells were kept in MEM supplemented with $5-10 \%$ fetal bovine serum, glutamine, and antibiotics. Transfections of $5 \times 10^{5}$ cells were carried out by the calcium phosphate co-precipitation method as described (Rethwilm et al., 1991). The transfection cocktail contained $5 \mu \mathrm{g}$ of indicator plasmid and $1 \mu \mathrm{g}$ of effector plasmid, either pHbel-1s or pHbel-1a. The DNA concentration was adjusted to $20 \mu \mathrm{g} / \mathrm{ml}$ with herring sperm DNA. Cells were harvested after $48 \mathrm{hr}$ by scraping and lysates were prepared as described (Rethwilm et al., 1991). The protein concentration of the lysates was measured with a commercial assay (Bio-Rad). The amount of protein assayed and the incubation time were kept constant within a series of tests but differed from series to series (as specified in the figure legends) to be in the linear range. Lysates were analyzed in a total volume of $200 \mu \mathrm{l} 250 \mathrm{mM}$ Tris- $\mathrm{HCl}$, $\mathrm{pH} 8.0$ containing $0.5 \mathrm{mM}$ acetyl-CoA (Sigma) and 0.1 $\mu \mathrm{Ci}$ of $\left[{ }^{14} \mathrm{C}\right]$ chiloramphenicol (Amersham) at $37^{\circ}$. Nonacetylated chloramphenicol was separated from acetylated forms by ascending thin-layer chromatography and the spots were quantitated with a Molecular Dynamics Phosphorlmager. For each construct 5-6 independent transfections with DNA from two preparations were performed. Variation of CAT values for a given construct was less than $20 \%$.

\section{RESULTS}

\section{Negative effect of R-U5 sequences on HFV gene expression}

Previous studies on the role of R-U5 sequences on HFV LTR directed gene expression have led to controversial results. While a moderate increase in activity has been reported for U5 and R-U5 deleted LTR constructs in one study (Rethwilm et al., 1991), no such effect was observed by others (Keller et al., 1991). For SFV-1 and SFV-3 a marked increase in reporter gene 
AAGCTTTCACATACTCAGTAGCTGTTTCACAATCAACAAAACAATGATGATGTAATCATAGGAAGTAGTTT

AAGCTTTCACATACTCAGTAGCTGTTT

AGCTTTCACATACTCAGTAGCTGTTTCACAATCAA

TCACAATCAACAAAACAATGATGATG

CAAAACAATGATGATGTAATCATAAGGAAGTAGTTT

TAATCATAAGGAAGTAGTTT
$-361 /-291$

OLIGO TJ

OLIGO so

OLIGO MM

OLIGO BG

OLIGO HH

\begin{tabular}{|c|c|c|}
\hline - TAF & $+\mathrm{TAF}$ & $\begin{array}{l}\text { fold } \\
\text { activation }\end{array}$ \\
\hline 2.7 & 8.3 & 3 \\
\hline 2.5 & 83.2 & 33 \\
\hline 3.0 & 26.9 & 9 \\
\hline 2.9 & 75.5 & 26 \\
\hline 2.9 & 56.9 & 20 \\
\hline 2.9 & 13.6 & 5 \\
\hline 3.2 & 23.6 & 7 \\
\hline 3.2 & 24.9 & 8 \\
\hline 3.1 & 11.6 & 4 \\
\hline 3.1 & 11.3 & 4 \\
\hline 3.0 & 18.3 & 6 \\
\hline
\end{tabular}

FIG. 6. BRE II is located between nucleotide positions (-361/-291). Sequences of the upper strands of the Hindlil/Dral (-361/-291) fragment of U3 spanning the BRE II and of chemically synthesized oligonucleotides that were used to dissect element II are shown. The arrows above the open boxes indicate the orientation of the oligonucleotides with respect to the direction of transcription. The filled boxes represent the longer DNA fragments introduced into pTATA. Assay conditions: $50 \mu \mathrm{g}$ protein was incubated for $60 \mathrm{~min}$.

expression of R-U5 deleted LTRs has been described (Mergia et al., 1992; Renne et al., 1993). We therefore wanted to analyze the effect of R-U5 on reporter gene expression by measuring the CAT activity of LTR mutants with nested deletions introduced from the $3^{\prime}$ end after co-transfection with pHbel-1s (Fig. 1). As shown in Fig. 2, up to 30-fold increase in TAF HFV mediated CAT gene expression was obtained with mutants deleting U5 (construct +195 ) compared to a construct that extends into the primer binding site (construct +351 ). Further deletions of $R$ sequences did not result in higher levels of expression. However, it is likely that 

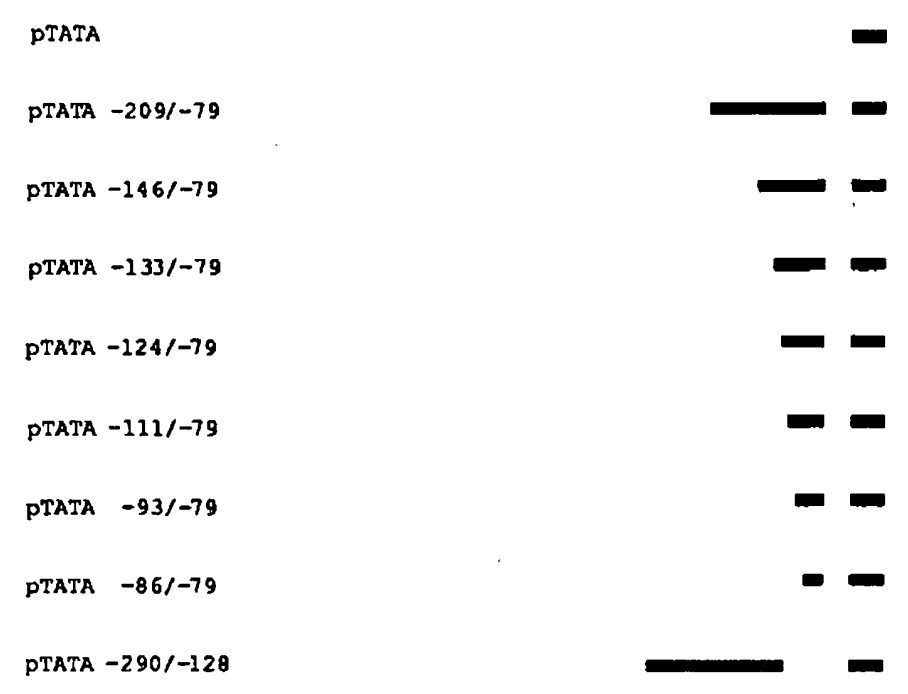

\begin{tabular}{|c|c|c|}
\hline- TAF & + TAF & $\begin{array}{c}\text { fold } \\
\text { activation }\end{array}$ \\
\hline 2.7 & 14.0 & 5 \\
\hline 0.8 & 80.0 & 100 \\
\hline 0.6 & 88.8 & 111 \\
\hline 1.1 & 78.3 & 71 \\
\hline 1.6 & 88.6 & 55 \\
\hline 1.5 & 53.3 & 36 \\
\hline 2.4 & 35.6 & 14 \\
\hline 2.5 & 23.9 & 10 \\
\hline 1.6 & 10.7 & 7 \\
\hline
\end{tabular}

FIG. 7. BRE III is located between nucleotide positions -124 and -93 . Nested 5 ' deletions of the fragment $-209 /-79$ introduced in the pTATA vector were assayed to map BRE III. Assay conditions: $50 \mu \mathrm{g}$ protein was incubated for $60 \mathrm{~min}$.

the assays with further $R$ truncations were beyond the linear range in this assay series. Interestingly, the viral cap site and adjacent $5^{\prime}$ sequences were not found to be required for $T A F_{\mathrm{HFV}}$ augmented gene expression (construct -13 in Fig. 2). A significant reduction in activity was only noted with construct -79 which deletes the HFV TATA box.

\section{Mapping of the BEL-1 responsive region}

To map $T A F_{H F V}$ responsive sequences in the $\mathrm{U} 3 \mathrm{re}$ gion of the HFV LTR we first investigated a set of nested $5^{\prime}$ deletions which are depicted in Fig. 3. An increase in activity was observed when the $5^{\prime} 280$ bp of U3 were deleted regardless of whether or not the constructs contained the R-U5 region. However, this effect was more pronounced with constructs bearing R-U5. Using the deletion mutants of both construct types, a first reduction in activity was found at position -261 and the second reduction was observed at position -93 . These results indicate that the major BEL-1 responsive region is located in $U 35^{\prime}$ of position -93 and that there is at least one further element which may be located upstream of position -261 .

Truncations of U3 from the $3^{\prime}$ end were then analyzed. To facilitate this an EcoRV restriction site was introduced by in vitro mutagenesis at position $-34 \mathrm{im}$ mediately $5^{\prime}$ to the TATA box. As shown in Fig. 4, the introduction of the EcoRV site does not significantly alter the activity of the HFV LTR, either with or without $\mathrm{R}-\mathrm{U} 5$, in response to the transactivator. Using the $3^{\prime}$ deletion mutants, the $5^{\prime}$ border of the BEL- 1 response region was determined to be located at approximately position -471 , since sequences extending from -471 to -777 did not respond to $T A F_{\mathrm{HFV}}$. These experiments also revealed two reductions in activity between positions -378 and -442 and between positions -442 and -471 indicating major responsive sequences downstream of positions -442 and -471 .

Taken together, the results obtained with the $5^{\prime}$ and $3^{\prime}$ deletion mutants of $\mathrm{U} 3$ show that the complete BRE is located between positions -93 and -471 relative to the start of transcription. There is also evidence for several target sites of $\mathrm{TAF}_{\mathrm{HFV}}$ within this element.

\section{Physical dissection of the BRE}

We next investigated to what extent portions of the BRE were able to respond to $T_{A F} F_{\mathrm{HFv}}$. To accomplish this, HFV U3 fragments were cloned into the pTATA vector which essentially bears only the core promoter sequences between positions -33 and +4 . PTATA itself, however, is not completely silent and is activated approximately two-to sixfold when co-transfected with the BEL-1 expression plasmid (Figs. 3-8). Thus, only those fragments that were stimulated significantly more than 6 fold were regarded as BEL-1 responsive.

The first sequence studied for $\mathrm{TAF}_{\mathrm{HFV}}$ responsiveness was the portion upstream of position -358 . Within this region the HFV LTR bears three recognition sequences for the ubiquitous transcription factor AP- 1 . It had been shown previously that AP-1 exerts a moderate effect on HFV promoter activity and is dependent on functionally active AP-1 binding sites. However, this effect was found to be distinct from the action of BEL-1 


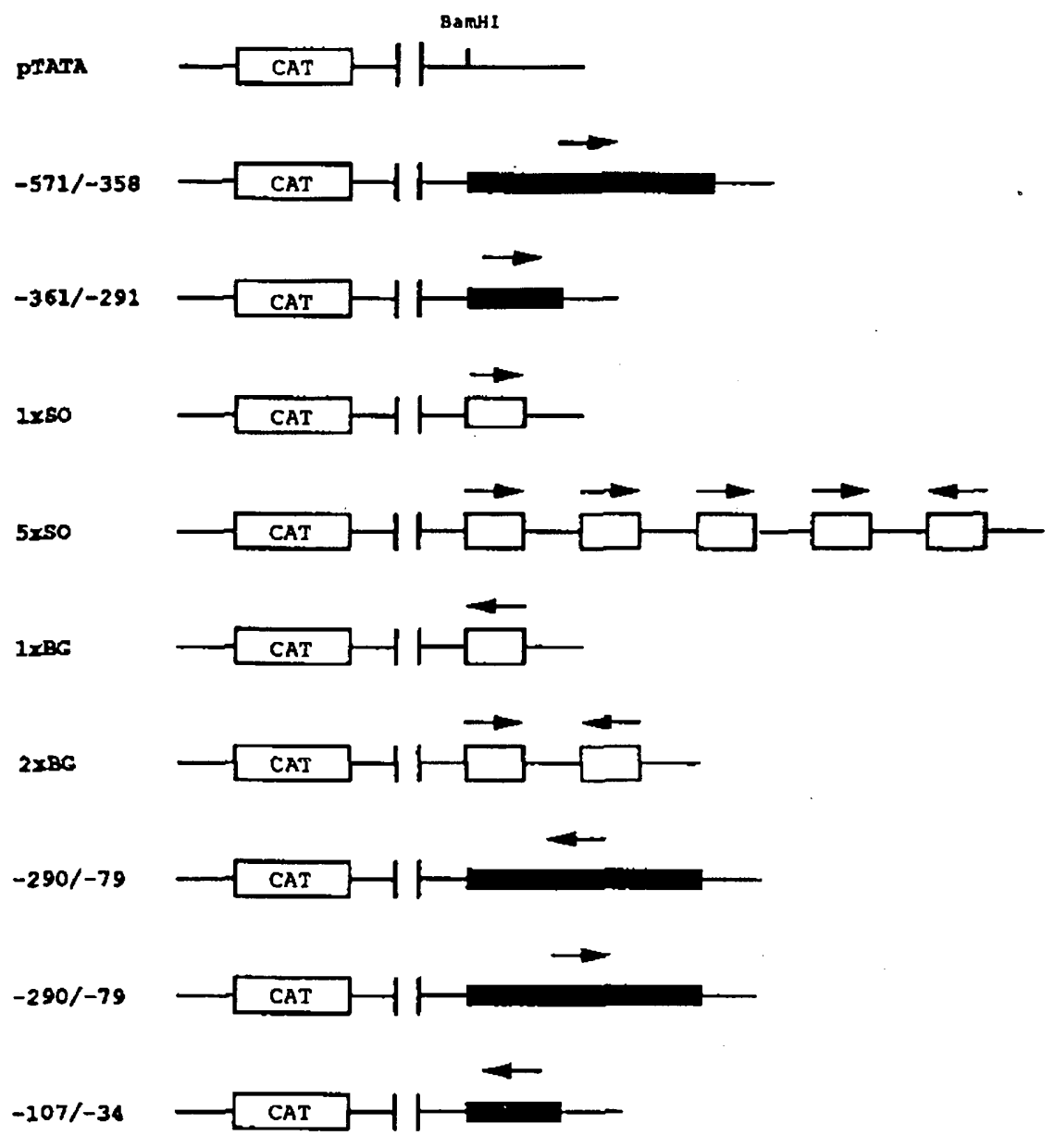

\begin{tabular}{|c|c|c|c|}
\hline BRE & - TAF & + TAF & $\begin{array}{c}\text { fold } \\
\text { activation }\end{array}$ \\
\hline & 1.6 & 9.6 & 6 \\
\hline$I+A P-1$ & 1.6 & 72.3 & 45 \\
\hline II & 1.7 & 31.6 & 18 \\
\hline & 1.2 & 24.4 & 20 \\
\hline & 1.3 & 56.1 & 43 \\
\hline & 1.6 & 15.7 & 10 \\
\hline & 1.5 & 9.7 & 6 \\
\hline II I & 1.2 & 6.6 & 6 \\
\hline III & 1.2 & 5.4 & 5 \\
\hline & 1.9 & 6.5 & 3 \\
\hline
\end{tabular}

FiG. 8. Analysis of positional effects of BRE I-III on enhancer activity. DNA tragments and oligonucleotides covering BRE I-III introduced into the BamHI site downstream of the CAT gene in the pTATA vector were measured for BEL-1 response. Filled boxes represent longer DNA fragments and open boxes represent oligonucleotides. The arrows indicate the orientation of the cloned sequences with respect to the CAT gene. Assay conditions: $50 \mu \mathrm{g}$ lysate was incubated for $60 \mathrm{~min}$.

(Maurer et al., 1991). When 5 ' truncations of the -534 / -358 fragment (Fig. 5) were analyzed, DNA fragments retained inducibility by $T_{A F} F_{\mathrm{HFV}}$ until truncations passed position -413 where the first reduction in activity was noticed. A more dramatic decrease in activity was observed between positions -399 and -397 which may indicate that a crucial DNA site for BEL-1 action was affected. Taken together the results from Figs. 4 and 5 , the BEL -1 target site I was duly identified to be located in the 36 bp region between positions -413 and -378 . The site therefore resides outside the AP-1 binding sites which are located upstream of position -439 . We also observed that the $5^{\prime}$ deletions analyzed in this experiment all gave rise to lower stimulative indices than the construct $-777 /-358$ in Fig. 4 that covers the same target element. This may be attributed to the lower basal activity of the latter.

The second portion to be studied was defined by the restriction sites for HindIII and Dral at positions -361 and -291 , respectively. When this fragment was placed in front of the CAT gene in the pTATA vector, a clear response to $\mathrm{TAF}_{\mathrm{HFV}}$ was noted (Fig. 6). Because of the relatively small size of this element, we attempted to further dissect the BEL-1 target site by using oligonucleotides that were introduced into the pTATA vector (Fig. 6). Oligonucleotide SO spanning the $5^{\prime}$ half of the HindllWDral tragment was activated approximately 20 -fold by $T A F_{\mathrm{HFV}}$, but this did not achieve the stimulative index of the complete fragment. The activation of the SO element was found to be orientation independent with respect to the direction of transcription. Oligonucleotide BG spanning the $3^{\prime}$ half of the Hindll//Dral fragment did not respond to TAF $_{\mathrm{HFV}}$ when a single copy was present in the construct. However, three copies of this element gave rise to a measurable response, irrespective of its orientation. This may indicate that the SO and $B G$ sequences are only partially able to fulfill the requirements of a 


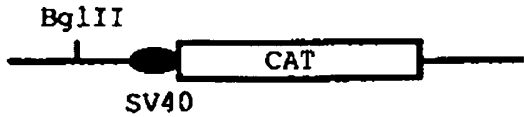

PA 10 CAT

$$
\begin{aligned}
& -777 /-79 \\
& -571 /-34 \\
& -361 /-34 \\
& -135 /-34 \\
& -107 /-34 \\
& -84 /-34 \\
& -290 /-79 \\
& -361 /-291 \\
& -571 /-358 \\
& -435 /-358 \\
& -399 /-358
\end{aligned}
$$

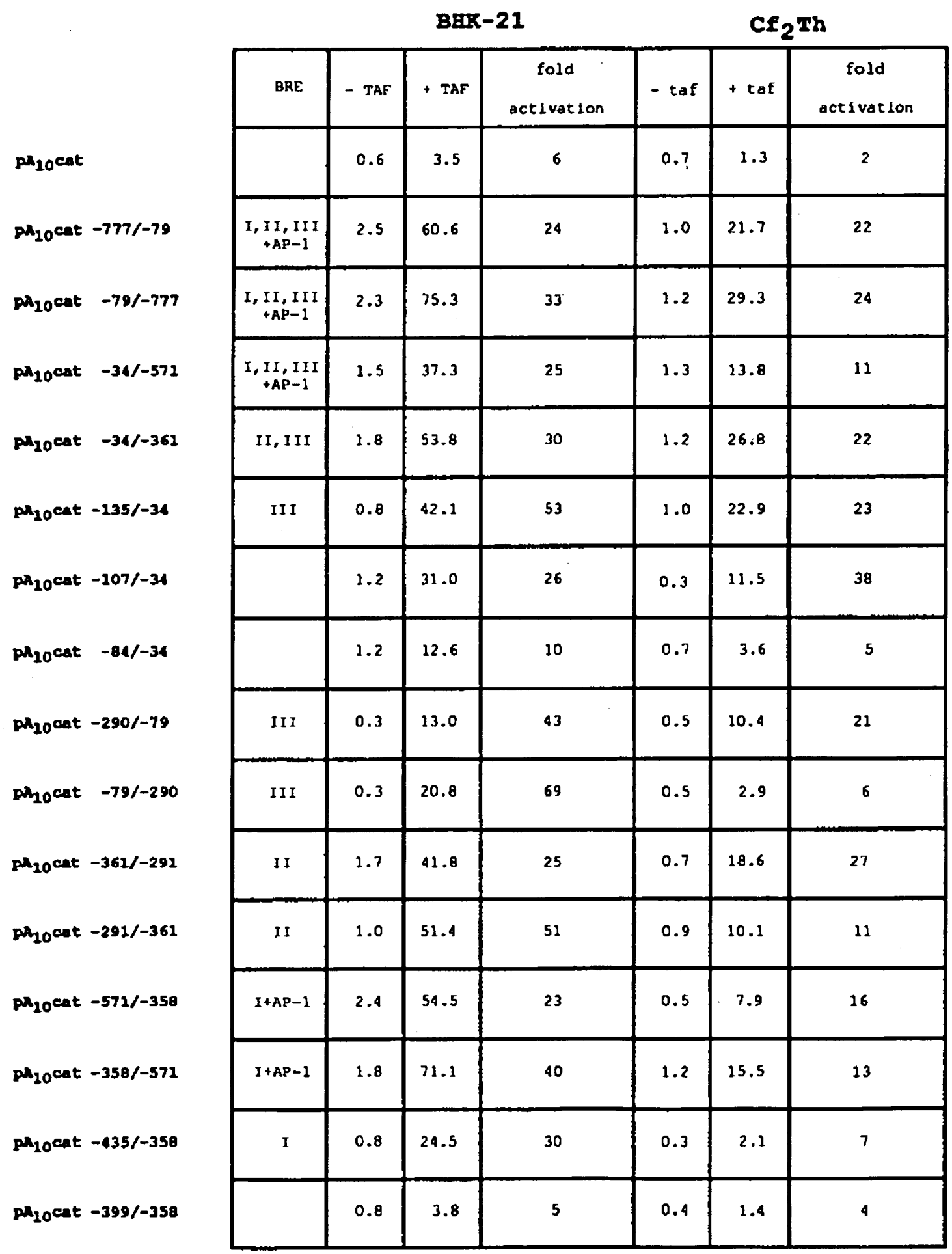


"NRE" AP-1

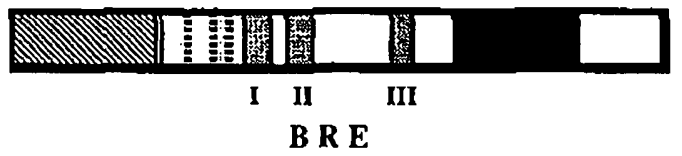

Fig. 10. Schematic drawing of the HFV LTR showing the cis-regulatory elements identified in this study. "NRE" represents a proposed weak negative regulatory element in the upstream U3 region, AP-1 marks the region with the three binding sites for the transcription factor AP-1, and BRE I to III indicate the three BEL-1 transactivator responsive elements.

functionally active BEL-1 target site. When subfragments of $S O$ and $B G(T J$ and $H H$ ) or an oligonucleotide spanning the middle of the Hindlll/Dral fragment and overlapping $S O$ and $B G(M M)$ were analyzed, none of these was significantly activated over background values, even when multimerized oligonucleotides were tested. In summary, these results suggest that the complete target site 11 is located within the 71 bp region between positions -361 and -291 . To show that elements I and II are indeed physically distinct, we also tested a DNA fragment that spans the overlap of both elements (construct $-384 /-345$ in Fig. 6). Consistent with the view of two target sites this element was not found to be stimulated by TAF $\mathrm{HFV}$.

The third portion analyzed was the promoter proximal element. As shown in Fig. 7, the region between -209 and -79 responded well to BEL-1. When nested $5^{t}$ deletions of this fragment were tested, CAT activity remained high for constructs with truncations up to position -124, then gradually declined and approached values close to background as the deletion reached position -93 . Together with the results shown in Fig. 3, target site III was localized within the 32 bp region between positions $-124 /-93$. We also studied the sequence between targets II and III using the construct $-290 /-128$ (Fig. 7) which did not respond to $T A F_{\mathrm{HFV}}$, indicating that there are no further $\mathrm{BEL}-1$ targets in this region. Interestingly, the -290 / -128 construct also covers the sequence of HFV which shows homology to an element in the HIV-1 U3 region and has been implicated in cross-transactivation of HIV-1 by HFV BEL-1 (Lee et al., 1992). The irresponsiveness of construct $-290 /-128$ to BEL- 1 indicates that the homologous sequence motif is not sufficient to act as a BEL-1 target.

To test whether or not positional effects influence the activity of the identified BREs, we cloned several fragments into the BamHI site of the pTATA vector approximately 1600 bp downstream of the promoter. The results of co-transfections using these constructs with pHbel-1s are shown in Fig. 8. Construct $-571 /-358$ which covers element $I$ and the three AP- 1 sites is activated to approximately the same extent as the -534 / -358 fragment inserted close to the reporter gene into the BgIll site (Fig. 5). Element II (construct -361/-291) is also activated when cloned downstream of the CAT gene, however, to a lesser extent compared to the respective fragment inserted into the Bg/ll site (Fig. 6). When the oligonucleotides SO and BG (Fig. 8) were substituted for the complete Hindlli/Dral fragment spanning element II, the BG element was found not to respond to $T A F_{H F V}$ whereas element SO appeared to be an active target site for the transactivator. The increase in reporter gene activity of a construct bearing multimerized copies of the SO sequence is typical for inducible enhancer elements (Serfling et al., 1985). In contrast to the findings with BRE I and II we found that element III did not respond significantly to $T_{A F} \mathrm{HFy}_{\mathrm{H}}$ when located at some distance from the promoter.

\section{The BEL-1 targets are conditional enhancer elements}

We finally investigated to what extent the BREs are active in a heterologous promoter context. HFV LTR portions, or oligonucleotides, were inserted into the SV40 promoter bearing CAT expression plasmid $p A_{10}$ cat that had been used previously to identity TAX responsive sequences in the LTR of human T-cell leukemia virus type I (HTLV-I) (Paskalis et al., 1986). And in addition to the BHK-21 cells exclusively used in the experiments reported above constructs were also analyzed in the C†2Th cell line.

The results of these experiments are summarized in Fig. 9. When U3 fragments covering all BREs were analyzed they were found to respond to BEL-1 irrespective of their orientation to the CAT gene in both cell lines tested. Furthermore, all but one individual elements that were previously found to confer BEL- 1 responsiveness to the HFV promoter were also found to be active with the SV40 promoter, although to a lesser extent. The $-399 /-358$ construct, partially active in the pTATA vector (Fig. 5), showed no response in the $\mathrm{pA}_{10}$ cat context. All three BREs were active independent of their orientation with respect to the reporter gene. However, some cell type differences were noticed. Generally, transactivation was found to be

FiG. 9. Analysis of the promoter and cell type dependence of BRE I-III. U3 DNA fragments covering BRE I-III were introduced into the 8gIII site of $\mathrm{PA}_{10}$ cat. The orientation of the fragments relative to the reporter gene can be deduced from the position of the numbers after each construct which indicate the fragment borders. The dotted lines represent the AP-1 binding sites. Assay conditions: $25 \mu 9$ protein of BHK cell lysates were incubated for $40 \mathrm{~min}$, and $50 \mu \mathrm{g}$ protein of Cf2Th cell lysates was incubated for $60 \mathrm{~min}$. 
weaker in dog thymus cells, in particular, construct $-435 /-358$ covering element $I$ and construct -79 / -290 bearing element $\mathrm{II}$ in reverse orientation with respect to the reporter gene, were barely stimulated.

\section{DISCUSSION}

We report here a detailed analysis on the BEL-1 responsive sequences in the LTR of HFV. This was facilitated by analyzing LTR fragments in both homologous and heterologous promoter contexts for responsiveness to the BEL- 1 transactivator.

The R-U5 region was found to negatively influence HFV LTR directed gene expression. The negative regulatory function of SFV-1 and SFV-3 R-U5 has been attributed to an extensive secondary structure of the nascent R-U5 mRNA (Mergia et al., 1992; Renne et al., 1993). Analysis of HFV R-U5 sequences, using the Genetics Computer Group Fold Program (University of Wisconsin, Madison, WI), revealed a secondary structure with the free energy of $-73 \mathrm{kcal} / \mathrm{mol}$ (data not shown). The significance of this finding for the observed effect of R-U5 on HFV gene expression remains in question until studies using in vitro mutagenized LTR constructs, which disrupt the postulated RNA secondary structure, have been performed. Furthermore, it is so far not clear whether co-transcriptional or post-transcriptional events, as for example the utilization of the HFV mRNA, are responsible for the observed negative effect of R-U5. However, it is noteworthy that the splice donor of HFV has been mapped to position +51 (Muranyi and Flügel, 1991). A putative negative effect of R-U5 would therefore influence the genomic or gag-pol mRNA alone. Improvement of the transactivation values obtained with the R-U5 deletion mutants presented in Fig. 2 seems not to be the result of splicing events using the HFV splice donor at position +51 , since the constructs extending to positions +195 showed similar activation levels as constructs that are lacking the splice donor ( +4 and +35 in Fig. 2).

A second element which exerts a weak negative effect on HFV LTR directed gene expression was located in the upstream $\mathrm{U} 3$ region. A similar observation has been described for the upstream U3 region of SFV-3 (Renne et al., 1993). A negative regulatory element has also been located in the U3 region of HIV-1 (Rosen et al., 1985). However, to what extent the HFV sequences constitute a true negative regulatory element remains to be determined, for example, by testing its effect on the activity of a variety of constitutive promoters.

Three elements in the HFV LTR were identified that were able to respond independently from each other to BEL-1. Element I was mapped to sequences -413 / -378 relative to the start of transcription. It resides outside the binding sites for the transcription factor
AP-1 which has previously been shown to exert a moderate effect on HFV gene expression (Maurer et al., 1991). While element I was found to be active with the homologous as well as with the SV4O promoter in hamster kidney cells, it was only weakly stimulated in dog thymus fibroblasts when placed in the heterologous promoter context. Whether or not this indicates any enhancer cell specificity (Serfling et al., 1985) might be shown by a closer analysis using additional cell lines and different promoters.

Element II was located between positions -361/ -291. Because element II responded to $T_{A F} F_{H F V}$, irrespective of its position and orientation relative to the promoter and irrespective of the kind of promoter used and cell type analyzed, it behaves like a typical inducible enhancer (Serfling et al., 1985). When fragments of element II were analyzed for enhancer activity (oligonucleotides SO and BG in Fig. 6) they gave rise to a partial response that could be restored by multimerization of the targets. Further subfragments were found to respond only weakly to BEL-1. The dissection of element II into functional partially active fragments may indicate that BEL-1-mediated transactivation is a complex mechanism which might involve DNA sites with different specificities. BREs I and II were found to lay close to each other. However, the inactivity of a DNA fragment partially overlapping both elements $(-384 /-345$ in Fig. 6) is indicative of two separate elements.

Element III was mapped to the positions -124/-93. This element was also found to be active in dog thymus cells with the heterologous promoter, irrespective of its orientation to the reporter gene (Fig. 9). However, it did not act well when placed distant from the promoter (Fig. 8). This might reflect the natural situation in the HFV LTR, where BRE III is located next to the promoter. Figure 10 summarizes the organization of the HFV LTR deduced from our studies.

In a recent study Lee et al. (1993) identified five BEL1 response elements in the HFV LTR that were located at positions $-559 /-506$ (a), $-454 /-418$ (b), $-360 /$ -342 (c), $-327 /-284$ (d), and $-116 /-89$ (e). While our mapping experiments are in close agreement with respect to the elements c-e we could not show that fragments covering the region of elements $a$ and $b$ in the study of Lee et al. contribute substantially to the BEL-1 mediated transcriptional activity of the HFV LTR.

The HFV BRES I-III do not share sequence homologies, and to our knowledge, do not show obvious homologies to known eukaryotic enhancer sequence motifs. In this respect HFV is similar to SFV type 1 and 3 (Mergia et al., 1992; Renne et al., 1993). In preliminary experiments we were unable to demonstrate direct binding of a prokaryotically expressed $\beta G A L / B E L-$ 1 fusion protein to HFV LTR sequences, although bacterially expressed $\beta$ GALJUN protein did bind to its target sequence (data not shown). Similar observa- 
tions have been made recently (Venkatesh et al., 1993). It is therefore likely that BEL-1 mediated transactivation makes use of a novel method to regulate retrovirus gene expression which might also involve, as yet, undiscovered cellular transcription factors. These cellular factors must be ubiquitous since replication and transactivation of HFV have been reported for a wide range of cells from different tissues and species (Hooks and Detrick-Hooks, 1981; Rethwilm et al., 1991; Keller et al., 1991). With respect to the likely involvement of cellular factors in transactivation by BEL-1, HFV is reminiscent of HTLV-I (Marriot et al., 1989; Fujisawa et al., 1991; Zhao and Giam, 1992; Tong-Starksen and Peterlin, 1990). The finding that a sequence distinct from the 21 bp repeat can serve as a target for TAX (Marriot et al., 1989) is a further analogy between HTLV -1 and the results reported here for the disparate elements of the HFV LTR responding to BEL-1.

The requirements for BEL-1-mediated transactivation have been analyzed at the protein level by using mutants of BEL-1 (Venkatesh et al., 1993; He et al., 1993). However, the results have been controversially interpreted. While Venkatesh et al. (1993) suggested the minimal activation domain to be located in the region of aa positions 56-227. He et al. (1993) mapped the activation domain to the $50 \mathrm{c}$-terminal aa of the 300 aa BEL-1 protein and favored the view of a direct interaction of BEL- 1 with its target DNA. This conclusion was mainly drawn from the activation of the human immunodeficiency virus type 1 LTR by chimeras comprising the VP16 activation domain and c-terminally truncated BEL-1.

Fundamental questions in elucidating the molecular mechanism of BEL-1 transactivation of its own LTR are still open, for example, whether or not BEL-1 interacts directly with the target DNAs, or perhaps has DNA binding properties itself, and what additional cellular proteins may be involved in BEL-1 transactivation. The knowledge of the exact target sites for BEL- 1 identified in this study will be of use in answering these questions.

\section{ACKNOWLEDGMENTS}

We thank Edgar Sertling and Lee Dunster for critical review of the manuscript, Dieter Neumann-Haefelin and Ivan Horak for the gift of reagents. Sandra Brautigam for comments on the manuscript and help with the computer analysis, and Volker ter Meulen for support. This work was supported by the Deutsche Forschungsgemeinschatt (SFB 165).

\section{REFERENCES}

Baunach, G., Maurer, B., Hahn, H., Kranz, M., and Rethwilm, A. (1993). Mutational analysis of human foamy virus accessory reading frames. J. Virol., in press.

CULLEN, B. R. \{1991). Human immunodeficiency virus as a prototypic complex retrovirus. J. Virol. 65, 1053-1056.
Fujisawa, J.-I., MASAMI, T., YOSHIMURA, T., and YOSHIDA, M. (1992). The indirect association of human T-cell leukemia virus tax protein with DNA results in transcriptional activation. J. Virol. 65, 45254528.

He, F., Sun, J. D., Garrett, E. D., and Cullen, B. R. (1993). Functional organization of the bel-1 trans-activator of human foamy virus. J. Virol. 67, 1896-1904.

Hooks, J. J., and Detrick-Hooks, B. (1981). Spumavirinae: Foamy virus group infections: Comparative aspects and diagnosis. In "Comparative Diagnosis of Viral Diseases" (E. Kurstak and C. Kurstak, Eds.), Vol. 4. pp. 599-618. Academic Press, San Diego.

Keller, A., Partin, K. M., Lochelt, M., Bannert, H., Flügel, R., and CULLEN, B. R. (1991). Characterization of the transcriptional trans activator of human foamy retrovirus. J. Virol. 65, 2589-2594.

Keller, A., Garrett. E. D., and Cullen, B. R. (1992). The bel-1 protein of human foamy virus activates human immunodeficiency virus type I gene expression via a novel DNA target site. J. Virol. 66, 3946-3949.

Kimura, A., ISRAEL, A., Le Bail, O., and Kourilsky, P. (1986). Detailed analysis of the mouse $\mathrm{H}-2 \mathrm{~K}^{\mathrm{D}}$ promoter: enhancer-like sequences and their roie in the regulation of class I gene expression. Cell 44, 261-272.

LAiMins, L. A., Gruss, P., PozzatTi, R., and Khoury, G. (1984). Characterization of enhancer elements in the long terminal repeat of Moloney murine leukemia virus. J. Virol. 49, 183-189.

LEE, A. H., LEE, K. J., KIM, S., and SUNG, Y. C. (1992). Transactivation of human immunodeficiency virus type 1 long terminal repeat-directed gene expression by the human foamy virus bel 1 protein requires a specific DNA sequence. J. Virol. 66, 3236-3240.

LEE, K. J., LEE, A. H., and SUNG, Y. C. (1993). Multiple positive and negative cis-acting elements that mediate transactivation by bell in the long terminal repeat of human foamy virus. J. Virol. 67. 2317-2326.

MaraioT, S. J., Boros, I., DUVALL, J. F., and Brady, J. N. (1989). Indirect binding of human T-cell leukemia virus type I tax, to a responsive element in the viral long terminal repeat. Mol. Cell Biol. 9, 4152-4160.

Maurer, B., Serfling, E., Ter Meulen, V., and Rethwilm, A. (1991). Transcription factor AP-1 modulates the activity of the human foamy virus long terminal repeat. J. Virol. 65, 6353-6357.

Mergia, A., Shaw, K. E. S., Pratt-Lowe, E., Barry, P. A., and Luciw. P. A. (1990). Simian foamy virus type 1 is a retrovirus which encodes a transcriptional transactivator. J. Virol. 64, 3598-3604.

Mergia, A., Shaw, K. E. S., Pratt-Lowe, E., Barry, P. A., and Luciw, P. A. (1991). Identification of the simian foamy virus transcriptional transactivator gene (taf). J. Virol. 65, 2903-2909.

mergia, A., Pratt-lowe, E., Shaw, K. E. S., Renshaw-Gegg, L. W., and LuCiw, P. A. (1992). Cis-acting regulatory regions in the long terminal repeat of simian foamy virus type 1. J. Virol. 66, 251-257.

MURANYI, W., and FLUGEL, R. M. (1991). Analysis of splicing patterns of human spumaretrovirus by polymerase chain reaction reveals complex RNA structures. J. Virol. 65, 727-735.

O'HARE, K., BENOIST, C., and BREATHNACHT, R. (1981). Transtormation of mouse fibroblasts to methotrexat resistance by a recombinant plasmid expressing a prokaryotic dihydrofolate reductase. Proc. Natl. Acad. Sci. USA 78, 1527-1531.

Paskalis, H., Felber, B. K., and Pavlakis, G. N. (1986). Cis-acting sequences responsible for the transcriptional activation of human T-cell leukemia virus type I constitute a conditional enhancer. Proc. Natl. Acad. Sci. USA 83, 6558-6562.

Renne, R., Fried, E., Schweizer, M., Fleps, U., Turek, R., and NeuMANN-HAEFELIN, D. (1992). Genomic organization and expression of simian foamy virus type 3 (SFV-3). Virology 186, 597-608.

Renne, R., Mergia, A., Renshaw-Gegg, L. W., Neumann-Haefelin, D., and LUCIW. P. A. (1993). Reguiatory elements in the long terminal repeat (LTR) of simian foamy virus type 3 (SFV-3). Virology 192 , 365-369.

Rethwilm, A., Baunach, G., Netzer, K.-O., Maurer, B., Borisch, B., 
and Ter Meulen, V. (1990). Infectious DNA of the human spumaretrovirus. Nucleic Acids Res. 18, 733-738.

Rethwilm, A., Erlwein, O., Baunach, G., Maurer, B., and Ter MeuLEN, V. (1991). The transcriptional transactivator of human foamy virus maps to the bel 1 genomic region. Proc. Natl. Acad. Sci. USA 88, $941-945$.

Rosen, C. A., SODroski, J. G., and Haseltine, W. A. (1985). The location of cis-acting regulatory sequences in the human T-cell lymphotropic virus type III (HTLV-III/LAV) long terminal repeat. Cell 41, 813-823.

Sambrook, J., Fritsch, E. F., and Maniatis, T. (1989). "Molecular Cloning: A Laboratory Manual." Cold Spring Harbor Laboratory, Cold Spring Harbor, NY.

SANGER, F., NiCKLEN, S., and Coulsen, A. R. (1977). DNA sequencing with chain terminating inhibitors. Proc. Natl. Acad. Sci. USA 74, 5463-5467.
Serfing, E., Jasin, M., and SchaffneR, W. (1985). Enhancers and eukaryotic gene transcription. Trends Genet. 1, 224-230.

Tong-Starksen, S., and Peterlin, B. M. (1990). Mechanism of retroviral transcriptional activation. Semin. Virol. 1, 215-227.

Venkatesh, L. K., Theodorakis, P. A., and Chinnadural, G. (1991). Distinct cis-acting regions in U3 regulate trans-activation of the human spumaretrovirus long terminal repeat by the viral bel 1 gene product. Nucleic Acids Res. 19, 3661-3666.

Venkatesh, L. K., Yang, C., Theodorakis, P. A., and Chinnadural, G. (1993). Functional dissection of the human spumaretrovirus transactivator identifies distinct classes of dominant-negative mutants. J. Virol. $67,161-169$.

ZHAO, L.-J., and GIAM, C.-Z. (1992). Human T-cell lymphotripic virus type I (HTLV-I) transcriptional activator, tax, enhances CREB binding to HTLV-1 21-base-pair repeats by protein-protein interaction. Proc. Natl. Acad. Sci. USA 89, 7070-7074. 